\title{
Psychological Distress and Health-Related Quality of Life Among Women with Breast Cancer: A Descriptive Cross-Sectional Study
}

Nitikorn Phoosuwan ( $\nabla$ nitikorn.phoosuwan@pubcare.uu.se )

Uppsala Universitet https://orcid.org/0000-0001-6372-9921

Pranee C. Lundberg

Uppsala Universitet

\section{Research Article}

Keywords: Anxiety, Depressive symptoms, Social support, Survivor, Women with breast cancer

Posted Date: August 23rd, 2021

DOI: https://doi.org/10.21203/rs.3.rs-153257/v2

License: (c) (1) This work is licensed under a Creative Commons Attribution 4.0 International License.

Read Full License

Version of Record: A version of this preprint was published at Supportive Care in Cancer on December 23rd, 2021. See the published version at https://doi.org/10.1007/s00520-021-06763-z. 


\section{Abstract}

Purpose Globally, breast cancer is commonly found among women, and Sweden has the largest number of new cases diagnosed. Treatment leads to increased survival of patients, but they are at risk to experience psychological distress, including anxiety and depressive symptoms, and decreased healthrelated quality of life (HRQoL). This study investigated relationship between psychological distress and HRQoL, and related factors among women with breast cancer in Sweden.

Methods This descriptive cross-sectional study was conducted in Sweden. A total of 481 women with breast cancer from a list of a regional cancer centre answered a questionnaire about sociodemographic factors, social support, psychological distress and HRQoL. Data were subjected to Pearson's correlation and linear regression analyzes.

Results Psychological distress was related to HRQoL in terms of body image, future perspective, side effects of systemic therapy, breast symptoms, arm symptoms, and hair loss. Lower age was likely to increase symptoms of anxiety, and undergone breast reconstruction was likely to increase symptoms of depression. Breast reconstruction and chemotherapy worsened body image, low support from partner decreased sexual functioning and enjoyment, and low support from physicians and nurses worsened future perspective, side effects of systemic therapy, breast symptoms and indignation about hair loss.

Conclusions Psychological distress were correlated with the HRQoL. Increased support from physicians, nurses, and partner may increase the HRQoL among women with breast cancer. Treatments of women with breast cancer, such as breast reconstruction and chemotherapy were factors that decreased the psychological distress and increased the HRQoL.

\section{Background}

Worldwide, cancer is a major cause of morbidity and mortality; about 15 million new cases were found in 2012 and eight million people died from diseases related to cancer [1]. Breast cancer is the most common type of cancer among women, and more than 12 percent of women are diagnosed with breast cancer [2]. In Europe, Sweden has had the highest incidence rate during recent years [3], with nearly 8,000 women diagnosed with breast cancer each year [4]. Although improved diagnosis and treatment lead to increased survival rate among patients with breast cancer [5], 1,400 Swedish women died with breast cancer as the cause of death in 2006 [4]. During the survival of women with breast cancer after diagnosis, they are in high risk to experience psychological distress $[6,7]$ and may have decreased health-related quality of life (HRQoL) [8].

Psychological distress is a state of emotional suffering commonly characterized by symptoms of depression and anxiety [9]. More than $25 \%$ of women with breast cancer suffer from anxiety and depressive symptoms $[7,10]$. They are more likely to have suicidal thoughts than the general population [11]. Some factors are related to the psychological distress among them. Rural and Christian women with breast cancer are associated with anxiety and depressive symptoms in women with breast cancer [7], and 
negative life changes and traits of anxiety after surgery are predictors [12]. Psychological distress may be a forerunner to mental, physical, and emotional exhaustion in a country with high incident rate of breast cancer like Sweden $[9,13]$. There is need of an investigation of factors that help to avoid mental, physical and emotional chaos in patients with breast cancer [9].

HRQoL refers to an individual's perception of his or her position in life, covering independence, physical, psychological and social relations, and environmental and spiritual dimensions [14]. HRQoL has been acknowledged as an important outcome for patients with cancer [15]. HRQoL among women with breast cancer is often poorer in comparison with women in the general population regarding social and emotional functioning $[16,17]$. Sociodemographic characteristics including low age, low education, financial problems and occupation, could be factors associated with low HRQoL $[10,13,18,19]$. Chemotherapy treatment, time since diagnosis and lack of support from family are also associated with HRQoL among women with breast cancer $[8,10,13,18]$. In addition, psychological distress and stress are correlated with HRQoL $[8,19,20]$. Although important, these factors are often neglected or underrecognized [21]. Therefore, psychological distress needs to be investigated along with the HRQoL among women with breast cancer [21]. This study aimed at investigating the relationship between psychological distress and the HRQoL, and how sociodemographic factors, treatment and support factors are related to psychological distress and HRQoL among women with breast cancer in Sweden.

\section{Materials And Methods}

\section{Study setting and design}

A descriptive cross-sectional study was carried out in three cities in Sweden: Uppsala, Gävle and Falun.

\section{Participants}

Based on the Regional Cancer Centre (RCC) in Uppsala and Örebro, registered women were invited to participate in the study. The inclusion criteria were: women who (1) had been diagnosed with breast cancer at least one year before data collection, (2) were at least 18 years old, (3) lived in Uppsala, Gävle or Falun, and (4) were willing to participate. Women who reported a history of mental disorder or dementia were excluded. In total, 481 out of 975 eligible women with breast cancer agreed to participate in the study.

\section{Instruments}

Use was made of a questionnaire containing four parts: (1) sociodemographic characteristics, (2) social support, (3) psychological distress, and (4) HRQoL. Sociodemographic characteristics concerned age, marital status, education, religion, belonging to a cultural/ethnic minority, having an underlying disease, duration of diagnosed breast cancer, methods of treatment (e.g., chemotherapy, radiotherapy, Herceptin, and hormone therapy). 
The part social support concerned six sources of information support, viz. physicians, nurses, the Internet, partner, family members and friends, and the patient's institution. It was created by PCL. Each source comprised nine questions, each of which gave a score of zero if the answer was "No" and one if the answer was "Yes". Therefore, each source could give a total score ranging from zero to nine, and a higher total score indicated more information support. This part had a Cronbach's alpha coefficient of 0.89 .

The part psychological distress comprised of anxiety and depressive symptoms. Anxiety and depressive symptoms were measured by use of the Hospital Anxiety and Depression Scale [22]. The scale had 14 items divided into 2 subscales; one measured anxiety (HADS-A) and the other measured depressive symptoms (HADS-D). Each subscale had seven items with a four-Likert scale. The total possible score for each subscale ranged from zero to 21 , and a higher score indicated more symptoms. HADS-A and HADSD had Cronbach's alpha coefficients of 0.89 and 0.84 , respectively, for Swedish women with breast cancer [13].

HRQoL was measured using the European Organization for Research and Treatment of Cancer Breast Cancer-Specific Quality of Life Questionnaire (QLQ-BR23) [23]. It is a disease-specific questionnaire with 23 questions, each of which had four options assigned by a number (not at all=1, a little=2, quite a bit=3, and very much=4). It assessed eight dimensions: body image (BRBI), sexual functioning (BRSEF), sexual enjoyment (BRSEE), future perspective (BRFU), side effects of systemic therapy (BRST), breast symptoms (BRBS), arm symptoms (BRAS), and indignation by hair loss (BRHL) [24]. All dimensions were transformed to 100-percent scores, and higher scores indicated lower quality of life. This questionnaire was translated to Swedish and tested before data collection among other breast cancer patients with acceptable Cronbach's alpha score in each sub-scale [13].

\section{Procedure}

The heads and nurses of clinics of surgery/oncology and plastic surgery in Uppsala, Gävle and Falun were informed about the study. The heads of the clinics gave permission to conduct the study, and the nurses understood the study and were able to answer questions if the participants asked. Thereafter, written information about the study and its purpose together with a consent letter and a questionnaire was sent by ordinary mail to the eligible women. They were assured of their anonymity and of confidentiality, and they were told that they could drop out at any time. The Declaration of Helsinki for medical research was fulfilled. The women who agreed to participate in the study signed a consent letter, responded to the questionnaire, and returned these documents in a stamped envelope. Women who did not wish to participate in the study returned the documents without filling in any information. A reminder was sent twice by post (after two weeks and one month) to women who had not returned the envelope in due time.

Directed Acyclic Graphs (DAGs) [25] were constructed based on previous studies in order to demonstrate what factors were associated with psychological distress [26 - 28], and with HRQoL [13]. See Figures 1a, and $b$. 


\section{Analyses}

We analyzed data using descriptive and inferential statistics. Descriptive statistics summarized sociodemographic characteristics, social support, HADS-A scores, HADS-D scores, and QLQ-BR23 scores for participants in terms such as frequency, mean, and standard deviation (SD). Inferential statistics applied Pearson's correlation and linear regression analyzes.

Pearson's correlation was used to determine the correlation between the scores of the HADS-A, HADS-D, and each dimension of QLQ-BR23. Whilst linear regression analyses were performed to determine relationships between sociodemographic factors, treatments, and social support factors, and outcome variables.

In multiple linear regression analyses, outcome variables were the scores of psychological distress, and QLQ-BR23, all of which were continuous variables. Sociodemographic and social support were considered independent variables. Age, duration of diagnosed breast cancer, and each information support were continuous variables. Dummy variables (categorization to zero and one) were marital status (married/lived together $=0$, the others $=1$ ), education (high school or above $=0$, secondary school/others $=1$ ), belonging to a cultural/ethnic minority ( $\mathrm{no}=0$, yes $=1$ ), having an underlying disease (no=0, yes $=1$ ), and methods of treatments ( $\mathrm{no}=0$, yes $=1$ ). Religion was excluded because of a low number in its sub-group. Assumptions were satisfied before the analyses (i.e. auto-correlation, multicollinearity, homoscedasticity, linearity, and multivariate normality). First, we inserted each independent variable into a simple linear regression analysis for each outcome variable. Significant independent variables from the simple analyses remained in multiple linear regression analyses using stepwise selection method (alpha-to-enter of 0.05 , alpha-to-remove of 0.10 ). We provided adjusted $\mathrm{R}^{2}$ and a standardized partial regression coefficient $(\beta)$ and $95 \%$ confidence interval $(\mathrm{Cl})$ to demonstrate the fitness and strength of association of each outcome variable. The level of statistical significance for all analyses was set at $p<0.05$.

\section{Results}

Mean age (and SD) of participants was 62.7 (12.35), while average number of year (and SD) from diagnosis was 2.9 (3.97). Approximately $60 \%$ of the participants had no underlying diseases, and $20 \%$ of them underwent breast reconstruction. See Table 1. 
Table 1

Number and percentage of sociodemographic and treatment characteristics among participants $(n=481)$

\section{Characteristics}

$\mathrm{n}(\%)$

Age (years) ${ }^{a}$

Mean $=62.7, \mathrm{SD}=12.35, \mathrm{Min}=31, \mathrm{Max}=93$

Civil status $^{\mathrm{a}}$

Married/ Live together

$321(67.1)$

Single/alone

157 (32.9)

Education level $^{a}$

Secondary school/unidentified

$226(47.4)$

High school or university

$251(52.6)$

Religion $^{\mathrm{a}}$

Christian

$422(90.4)$

Non-Christian

$45(9.6)$

Cultural/Ethnic minority ${ }^{a}$

No

$455(96.8)$

Yes

$15(3.2)$

Underlying diseases ${ }^{a}$

No

277 (59.3)

Yes

$190(40.7)$

Duration from diagnosis (year) ${ }^{a}$

Mean $=2.92, \mathrm{SD}=3.97, \operatorname{Min}=0.2, \operatorname{Max}=44$

Chemotherapy $^{\mathrm{a}}$

Yes

$234(49.6)$

No

$238(50.4)$

Radiation therapy ${ }^{a}$

Yes

$227(52.6)$

a obtained number < 481; Hospital Anxiety and Depression Scale - Anxiety sub-scale (HADS-A);

Hospital Anxiety and Depression Scale - Depression sub-scale (HADS-D). 


\section{Characteristics}

No

Hormone therapy ${ }^{\mathrm{a}}$

Yes

No

Herceptin treatment ${ }^{a}$

Yes

No

Breast reconstruction $^{a}$

Yes

No

Social support - physicians ${ }^{\mathrm{a}}$

Mean $=6.36, S D=2.78, \operatorname{Min}=0, \operatorname{Max}=9$

Social support - nurses ${ }^{a}$

Mean $=4.60, S D=3.22, \operatorname{Min}=0, \operatorname{Max}=9$

Social support - internet ${ }^{a}$

Mean $=0.75, S D=1.71, \operatorname{Min}=0, \operatorname{Max}=9$

Social support - partner ${ }^{a}$

Mean $=0.79, S D=1.65, \operatorname{Min}=0, \operatorname{Max}=9$

Social support - family and friends ${ }^{a}$

Mean $=0.77, S D=1.56, \operatorname{Min}=0, \operatorname{Max}=9$

Social support - patient institution ${ }^{\mathrm{a}}$

Mean $=0.79, S D=1.65, \operatorname{Min}=0, \operatorname{Max}=9$

HADS-A ${ }^{a}$

Mean $=7.07, S D=3.38, \operatorname{Min}=2, \operatorname{Max}=19$

HADS-D

a obtained number < 481; Hospital Anxiety and Depression Scale - Anxiety sub-scale (HADS-A); Hospital Anxiety and Depression Scale - Depression sub-scale (HADS-D). $\mathrm{n}(\%)$

$252(47.4)$

$293(62.7)$

$174(37.3)$

$82(18.7)$

$357(81.3)$

$93(19.7)$

$380(80.3)$ 


\section{Characteristics}

$\mathrm{n}(\%)$

Mean $=14.10, S D=2.14, \operatorname{Min}=7, \operatorname{Max}=19$

a obtained number < 481; Hospital Anxiety and Depression Scale - Anxiety sub-scale (HADS-A); Hospital Anxiety and Depression Scale - Depression sub-scale (HADS-D).

The correlation analyses demonstrated that the scores of HADS-A correlated significantly with all dimensions of the HRQoL except BRSEF and BRSEE, while HADS-D correlated significantly with all dimensions of the HRQoL. HADS-A had its strongest correlation 0.619 with BRFU, while HADS-D had its strongest correlation 0.325 with BRBI. See Table 2 .

Table 2

Correlation between the scores of the Hospital Anxiety and Depression Scale - Anxiety subscale (HADS-A) and Depression sub-scale (HADS-D) and all dimensions of the Breast CancerSpecific Quality of Life Questionnaire.

\begin{tabular}{|c|c|c|c|c|c|c|c|c|}
\hline Variables & BRBI & BRSEF & BRSEE & BRFU & BRST & BRBS & BRAS & BRHL \\
\hline HADS-A & $0.490 *$ & 0.067 & 0.130 & $0.619 *$ & $0.428^{*}$ & $0.330 *$ & $0.276^{\star}$ & 0.171 * \\
\hline HADS-D & $0.325^{\star}$ & $0.159 *$ & $0.280^{\star}$ & $0.316^{*}$ & $0.269 *$ & $0.256^{*}$ & $0.177 *$ & $0.104^{*}$ \\
\hline
\end{tabular}

According to Table 3 , breast cancer patients who were younger $(\beta=-0.230,95 \% \mathrm{Cl}-0.180,-0.279, p<.001)$, had an underlying disease $(\beta=0.219,95 \% \mathrm{Cl} 0.118,0.319, p<.001)$ and had received less information support from physicians $(\beta=-0.142,95 \% \mathrm{Cl}-0.212,-0.071, p=.003)$ were likely to get increased symptoms of anxiety. Those who had an underlying disease $(\beta=0.116,95 \% \mathrm{Cl} 0.072,0.163, \mathrm{p}=.015)$ and had undergone breast reconstruction ( $\beta=0.116,95 \% \mathrm{Cl} 0.061,0.182, p=.013$ ) were likely to get increased symptoms of depression. 
Table 3

Multivariate linear regression analysis results of the scores of the HADS-A, HADS-D, and HRQoL

\begin{tabular}{|c|c|c|c|c|c|c|}
\hline \multirow[t]{2}{*}{ Variables } & \multicolumn{3}{|c|}{ Unstandardized Coefficients } & \multicolumn{3}{|l|}{$\begin{array}{l}\text { Standardized } \\
\text { Coefficients }\end{array}$} \\
\hline & B & $\begin{array}{l}\text { Standard } \\
\text { error }\end{array}$ & $95 \% \mathrm{Cl}$ & $\begin{array}{l}\text { Beta } \\
\text { (Descending) }\end{array}$ & $\mathrm{t}$ & $\mathrm{p}$ \\
\hline \multicolumn{7}{|l|}{ HADS-A ${ }^{a}$} \\
\hline Constant & 9.447 & 1.008 & $\begin{array}{l}7.466 \\
11.428\end{array}$ & & 9.374 & $<.001 *$ \\
\hline Age & -0.065 & .014 & $\begin{array}{l}-.092 \\
-.038\end{array}$ & -0.230 & -4.692 & $<.001^{\star}$ \\
\hline $\begin{array}{l}\text { Having an underlying } \\
\text { disease }\end{array}$ & 1.526 & .332 & $\begin{array}{l}.874 \\
2.178\end{array}$ & 0.219 & 4.599 & $<.001^{\star}$ \\
\hline $\begin{array}{l}\text { Social support - } \\
\text { physicians }\end{array}$ & -0.175 & .059 & $\begin{array}{l}-.291 \\
-.059\end{array}$ & -0.142 & -2.965 & $.003^{\star}$ \\
\hline
\end{tabular}

\section{HADS-D}

\begin{tabular}{lccllll} 
Constant & 14.443 & 0.143 & $\begin{array}{l}14.162, \\
14.723\end{array}$ & & 101.289 & $<.001^{*}$ \\
\hline Breast reconstruction & 0.626 & 0.252 & $\begin{array}{l}.130, \\
1.121\end{array}$ & 0.116 & 2.482 & $.013^{*}$ \\
$\begin{array}{l}\text { Having an underlying } \\
\text { disease }\end{array}$ & 0.501 & 0.115 & $\begin{array}{l}.099, \\
.903\end{array}$ & 0.115 & 2.451 & $.015^{\star}$
\end{tabular}

\section{$\mathrm{BRB}^{\mathrm{a}}$}

\begin{tabular}{|c|c|c|c|c|c|c|}
\hline Constant & 59.467 & 6.742 & $\begin{array}{l}46.211 \\
72.722\end{array}$ & & 8.820 & $<.001 *$ \\
\hline Chemotherapy & 8.027 & 2.301 & $\begin{array}{l}3.503 \\
12.552\end{array}$ & 0.180 & 3.488 & $.001 *$ \\
\hline $\begin{array}{l}\text { Having an underlying } \\
\text { disease }\end{array}$ & 7.761 & 2.278 & $\begin{array}{l}3.283 \\
12.239\end{array}$ & 0.169 & 3.407 & $.001^{\star}$ \\
\hline Age & -0.303 & .099 & $\begin{array}{l}-.498 \\
-.108\end{array}$ & -0.166 & -3.059 & $.002^{\star}$ \\
\hline Breast reconstruction & 5.589 & 2.758 & $\begin{array}{l}.166 \\
11.011\end{array}$ & 0.102 & 2.026 & $.043 *$ \\
\hline \multicolumn{7}{|l|}{ BRSEF $^{\mathrm{a}}$} \\
\hline Constant & 57.389 & 4.227 & $\begin{array}{l}49.079 \\
65.699\end{array}$ & & 5.987 & $<.001 *$ \\
\hline
\end{tabular}




\begin{tabular}{|c|c|c|c|c|c|c|}
\hline \multirow{2}{*}{$\begin{array}{l}\text { Variables } \\
\text { Age }\end{array}$} & \multicolumn{3}{|c|}{ Unstandardized Coefficients } & \multirow{2}{*}{$\begin{array}{l}\begin{array}{l}\text { Standardized } \\
\text { Coefficients }\end{array} \\
0.281\end{array}$} & \multirow[b]{2}{*}{5.987} & \multirow[b]{2}{*}{$<.001 *$} \\
\hline & 0.403 & .067 & $\begin{array}{l}.271 \\
.536\end{array}$ & & & \\
\hline $\begin{array}{l}\text { Having an underlying } \\
\text { disease }\end{array}$ & 5.530 & 1.643 & $\begin{array}{l}2.299 \\
8.760\end{array}$ & 0.156 & 3.365 & $.001 *$ \\
\hline $\begin{array}{l}\text { Social support - } \\
\text { partner }\end{array}$ & -1.532 & .490 & $\begin{array}{l}-2.494 \\
-.569\end{array}$ & -0.143 & -3.129 & $.002 *$ \\
\hline $\begin{array}{l}\text { Belonging to a } \\
\text { culture/ethnic minority }\end{array}$ & 13.531 & 4.694 & $\begin{array}{l}4.303 \\
22.759\end{array}$ & 0.131 & 2.882 & $.004^{\star}$ \\
\hline \multicolumn{7}{|l|}{ BRSEE $^{\mathrm{a}}$} \\
\hline Constant & 25.446 & 8.902 & $\begin{array}{l}7.885 \\
43.006\end{array}$ & & 2.859 & $.005^{\star}$ \\
\hline Age & .535 & 0.161 & $\begin{array}{l}.217 \\
.853\end{array}$ & 0.247 & 3.316 & $.001 *$ \\
\hline Education & 9.005 & 3.521 & $\begin{array}{l}2.059 \\
15.951\end{array}$ & 0.191 & 2.558 & $.011 *$ \\
\hline $\begin{array}{l}\text { Social support - } \\
\text { partner }\end{array}$ & -1.703 & .789 & $\begin{array}{l}-3.259 \\
-.147\end{array}$ & -0.145 & -2.159 & $.032 \star$ \\
\hline \multicolumn{7}{|l|}{ BRFUa $^{a}$} \\
\hline Constant & 77.786 & 7.304 & $\begin{array}{l}\text { 63.428, } \\
92.144\end{array}$ & & 10.649 & $<.001 *$ \\
\hline Age & -0.548 & 0.097 & $\begin{array}{l}-0.739 \\
-0.357\end{array}$ & -0.272 & -5.639 & $<.001 *$ \\
\hline $\begin{array}{l}\text { Having an underlying } \\
\text { disease }\end{array}$ & 11.497 & 2.251 & $\begin{array}{l}\text { 7.072, } \\
15.922\end{array}$ & 0.230 & 5.107 & $<.001 *$ \\
\hline $\begin{array}{l}\text { Social support - } \\
\text { physicians }\end{array}$ & -1.503 & .402 & $\begin{array}{l}-2.293 \\
-.713\end{array}$ & -0.171 & -3.741 & $<.001^{*}$ \\
\hline Chemotherapy & 6.229 & 2.291 & $\begin{array}{l}1.726 \\
10.732\end{array}$ & 0.127 & 2.719 & $.007 *$ \\
\hline \multicolumn{7}{|l|}{ BRST $^{a}$} \\
\hline Constant & 28.431 & 1.578 & $\begin{array}{l}25.328 \\
31.533\end{array}$ & & 18.012 & $<.001 *$ \\
\hline $\begin{array}{l}\text { Having an underlying } \\
\text { disease }\end{array}$ & 8.391 & 1.180 & $\begin{array}{l}6.071 \\
10.711\end{array}$ & 0.316 & 7.110 & $<.001 *$ \\
\hline Chemotherapy & 5.118 & 1.174 & $\begin{array}{l}2.811 \\
7.424\end{array}$ & 0.197 & 4.361 & $<.001 *$ \\
\hline
\end{tabular}




\begin{tabular}{|c|c|c|c|c|c|c|}
\hline \multirow{2}{*}{$\begin{array}{l}\text { Variables } \\
\begin{array}{l}\text { Social support - family } \\
\text { and friend }\end{array}\end{array}$} & \multicolumn{3}{|c|}{ Unstandardized Coefficients } & \multirow{2}{*}{$\begin{array}{l}\text { Standardized } \\
\text { Coefficients } \\
-0.135\end{array}$} & \multirow[b]{2}{*}{-3.040} & \multirow[b]{2}{*}{$.003^{*}$} \\
\hline & -1.111 & .366 & $\begin{array}{l}-1.830 \\
-.393\end{array}$ & & & \\
\hline $\begin{array}{l}\text { Social support - } \\
\text { physicians }\end{array}$ & -.631 & .212 & $\begin{array}{l}-1.048 \\
-.215\end{array}$ & -0.135 & -2.979 & $.003^{\star}$ \\
\hline \multicolumn{7}{|l|}{ BRBS $^{a}$} \\
\hline Constant & 43.823 & 3.785 & $\begin{array}{l}36.383 \\
51.262\end{array}$ & & 11.579 & $<.001 *$ \\
\hline Radiotherapy & 7.040 & 1.255 & $\begin{array}{l}4.572, \\
9.507\end{array}$ & 0.252 & 5.607 & $<.001 *$ \\
\hline $\begin{array}{l}\text { Belonging to a } \\
\text { culture/ethnic minority }\end{array}$ & 16.237 & 3.718 & $\begin{array}{l}\text { 8.928, } \\
23.545\end{array}$ & 0.195 & 4.367 & $<.001 *$ \\
\hline Age & -0.226 & 0.054 & $\begin{array}{l}-0.332 \\
-0.119\end{array}$ & -0.196 & -4.159 & $<.001 *$ \\
\hline $\begin{array}{l}\text { Having an underlying } \\
\text { disease }\end{array}$ & 4.680 & 1.304 & $\begin{array}{l}2.118 \\
7.242\end{array}$ & 0.164 & 3.590 & $.018 *$ \\
\hline $\begin{array}{l}\text { Social support - } \\
\text { nurses }\end{array}$ & -.477 & .200 & $\begin{array}{l}-.870 \\
-.084\end{array}$ & -0.110 & -2.384 & $.018 *$ \\
\hline \multicolumn{7}{|l|}{ BRAS $^{a}$} \\
\hline Constant & 28.234 & 1.265 & $\begin{array}{l}\text { 25.746, } \\
30.721\end{array}$ & & 22.311 & $<.001 *$ \\
\hline Radiotherapy & 8.455 & 1.661 & $\begin{array}{l}5.190 \\
11.721\end{array}$ & 0.265 & 5.090 & $<.001 *$ \\
\hline $\begin{array}{l}\text { Having an underlying } \\
\text { disease }\end{array}$ & 8.462 & 1.457 & $\begin{array}{l}5.597 \\
11.326\end{array}$ & 0.260 & 5.808 & $<.001 *$ \\
\hline Chemotherapy & 3.991 & 1.655 & $\begin{array}{l}0.738 \\
7.244\end{array}$ & 0.125 & 2.411 & $.016 *$ \\
\hline \multicolumn{7}{|l|}{ BRHL } \\
\hline Constant & 32.794 & 1.879 & $\begin{array}{l}29,100 \\
36.487\end{array}$ & & 17.449 & $<.001^{*}$ \\
\hline $\begin{array}{l}\text { Social support - } \\
\text { physicians }\end{array}$ & -.706 & .271 & $\begin{array}{l}-1.238 \\
-.174\end{array}$ & -0.123 & -2.608 & $.009 *$ \\
\hline $\begin{array}{l}\text { Belonging to a } \\
\text { culture/ethnic minority }\end{array}$ & 10.450 & 4.430 & $\begin{array}{l}1.744 \\
19.157\end{array}$ & 0.111 & 2.359 & $.019 *$ \\
\hline
\end{tabular}




\section{Variables \\ Unstandardized Coefficients \\ Standardized \\ Coefficients}

a Chemotherapy and social support - nurses were significant only in univariate analyses for HADS-A; Radiotherapy Herceptin, social support - physicians, and social support - nurses were significant only univariate analyses for quality of life - body image. Breast reconstruction, civil status, education and social support - physicians were significant only in univariate analyses for BRSEF.

Chemotherapy and social support - internet were significant only in univariate analyses for BRSEE. Civil status and social support - nurses were significant only in univariate analyses for BRFU. Age was significant only in univariate analysis for BRST. Chemotherapy, civil status, and social support physicians were significant only in univariate analyses for BRBS. Age and social support - physicians were significant only in univariate analyses for BRAS.

HADS-A, $F=17.551, P<.001$, Adjusted $R^{2}=0.107$

HADS-D, $F=5.585, P=.004$, Adjusted $R^{2}=0.020$

$\mathrm{BRBI}, \mathrm{F}=11.067, \mathrm{P}<.001$, Adjusted $\mathrm{R}^{2}=0.093$

BRSEF, $F=21.160, P<.001$, Adjusted $R^{2}=0.164$

BRSEE, $F=11.775, P<.001$, Adjusted $R^{2}=0.146$

BRFU, $F=24.916, P<.001$, Adjusted $R^{2}=0.184$

BRST, $F=23.228, P<.001$, Adjusted $R^{2}=0.173$

BRBS, $F=19.798, P<.001$, Adjusted $R^{2}=0.185$

BRAS, $F=27.954, P<.001$, Adjusted $R^{2}=0.160$

$B R H L, F=6.377, P=.002$, Adjusted $R^{2}=0.024$

Hospital, Anxiety, and Depression Scale - Anxiety subscale (HADS-A); Hospital, Anxiety, and Depression Scale - Depression subscale (HADS-D); Health-Related Quality of Life (HRQoL); Quality of life - body image (BRBI); Quality of life - sexual functioning (BRSEF); Quality of life - sexual enjoyment (BRSEE); Quality of life - future perspective (BRFU); Quality of life - systemic therapy side effects (BRST); Quality of life - breast symptoms (BRBS); Quality of life - arm symptoms (BRAS); and Quality of life - upset by hair loss (BRHL).

Patients with breast cancer who had been treated with chemotherapy $(\beta=0.180,95 \% \mathrm{Cl} 0.145,0.215, p$ $=.001)$ and had an underlying disease $(\beta=0.169,95 \% \mathrm{Cl} 0.136,0.202, p=.001)$, were younger $(\beta=-0.166$, $95 \% \mathrm{Cl}-0.265,-0.067, \mathrm{p}=.002$ ) were associated with decreased HRQoL in BRBI. Patients who were older $(\beta=0.403,95 \% \mathrm{Cl} 0.336,0.470, p<.001)$ and had an underlying disease $(\beta=0.156,95 \% \mathrm{Cl} 0.068,0.244, p$ $=.001)$ were associated with decreased HRQoL in BRSEF. Patients who were older $(\beta=0.247,95 \% \mathrm{Cl}$ $0.162,0.332, p=.001)$ and had low education $(\beta=0.191,95 \% \mathrm{Cl} 0.090,0.284, p=.011)$ were associated with decreased HRQoL in BRSEE. Patients who were younger $(\beta=-0.272,95 \% \mathrm{Cl}-0.369,-0.175, \mathrm{p}<.001)$ and had an underlying disease $(\beta=0.230,95 \% \mathrm{Cl} 0.196,0.262, p<.001)$ were associated with decreased HRQoL in BRFU. Having an underlying disease $(\beta=0.316,95 \% \mathrm{Cl} 0.282,0.402, p<.001)$ and having 
undergone treatment with chemotherapy $(\beta=0.197,95 \% \mathrm{Cl} 0.169,0.232, \mathrm{p}<.001)$ decreased HRQoL in BRST. Having received radiotherapy $(\beta=0.252,95 \% \mathrm{Cl} 0.167,0.336, p<.001)$ and belonging to an ethnic minority $(\beta=0.195,95 \% \mathrm{Cl} 0.095,0.289, \mathrm{p}<.001)$ decreased HRQoL in BRBS. Having received radiotherapy $(\beta=0.265,95 \% \mathrm{Cl} 0.180,0.348, p<.001)$ and having an underlying disease $(\beta=0.260,95 \%$ $\mathrm{Cl} 0.174,0.342, \mathrm{p}<.001)$ were factors associated with decreased HRQoL in BRAS. Patients who had received increased social support from physicians $(\beta=0.123,95 \% \mathrm{Cl} 0.108,0.138, p=.009)$ and belonged to an ethnic minority $(\beta=0.111,95 \% \mathrm{Cl} 0.096,0.127, p=.019)$ were associated with decreased HRQoL in BRHL.

\section{Discussion}

In this study, both anxiety and depressive symptoms demonstrated the highest correlation with HRQoL in the dimensions of future perspective and body image. Psychological distress is commonly diagnosed among patients with breast cancer $[7,29]$. People living with psychological distress may experience imbalance between their realities and their ideal wishes, resulting in a break-down in their self-esteem and low well-being [9]. Moreover, psychological distress is recognized as associated with decreased HRQoL among patients [30]. Greater depressive symptoms are associated with more emotional suppression [12], and suicidal thoughts, and attempted suicide may occur among women with breast cancer suffering from depressive symptoms [11]. It might be useful to examine psychological distress and HRQoL along with treatment of psychologically vulnerable women, like women with breast cancer.

Our study revealed that participants who had an underlying disease were more likely to have psychological distress. Moreover, those having breast reconstruction might have more symptoms of psychological distress. People with psychological distress seem to have reduced capacity and lack of control of their everyday lives [9]. Although having an underlying disease is common, co-morbidity can make life difficult for patients with breast cancer [31]. Also, after life change events like surgery, patients with breast cancer may experience psychological distress [12]. Therefore, a preventive intervention related to emotional awareness for such patients with breast cancer should be implemented.

External sources, like information support from healthcare professionals (HCPs), could reduce psychological distress [32], while poor support contributes to psychological distress [33]. Development of treatment plans by physicians and patients is essential [34]. Patients need to be involved in a personcentered dialogue with physicians to strengthen their own capacities for daily live [9]. Therefore, our study suggests that HCPs should provide sufficient information to patients with breast cancer and include the patients in their planning. This may contribute to decreased psychological distress.

Several sources of information support (e.g. physicians, nurses, and partner) have been found to be related to decreased HRQoL for many dimensions. The participants needed support from their partners about their sexual functioning and enjoyment. After treatment of breast cancer, sexual dysfunction becomes a challenge for patients [34]. They need a more support and tenderness from their partners [31] to maintain their HRQoL [34]. Patients with breast cancer in our study also thought about side effects of 
their therapy, e.g., breast symptoms and hair loss. They needed information from HCPs about their current and future lives resulting in their increased well-being. Cognitive behavioral therapy and supportive-expressive group therapy give positive effects on patients with breast cancer [35]. Thus, after treatments of patients with breast cancer, individual or group therapy may assist improvement of the HRQoL among the patients.

\section{Strengths and limitations}

We constructed the DAGs from reviewed literature, which assisted data collection and analyses. Some confounders, such as age, had been adjusted by multiple linear regression analyses. The use of real scores from the questionnaire rather than categorized scores enhanced estimates [36]. In addition, all instruments used in this study had been tested before data collection with acceptable values of validity and reliability.

The cross-sectional character of the study limited cause-effect relationships. Therefore, subsequent longitudinal studies may more clearly explain factors associated with psychological distress and health related quality of life. Some information bias could be seen because the participants self-reported. In addition, the participation rate was $60 \%$ which may also have affected our findings.

\section{Conclusions}

Psychological distress was correlated with most dimensions of HRQoL. The strongest correlation was found for anxiety symptoms and future perspective, and depressive symptoms and body image. Women with breast cancer who were younger were likely to get increased symptoms of anxiety, while those who had undergone breast reconstruction were likely to get increased symptoms of depression. Low information support from HCPs decreased the HRQoL in terms of future perspective, systemic therapy side effects, breast symptoms, and indignation about hair loss. Support from partner increased the HRQoL in terms of sexual functioning and enjoyment. Treatment with chemotherapy decreased the HRQoL in terms of body image, systemic therapy side effects, and arm symptoms. Women with breast cancer are in need of social support from many sources, in particular HCPs and their partners.

\section{Declarations}

\section{Acknowledgments}

The authors thank all women with breast cancer who participated in this study.

\section{Funding}

This study was supported by the Breast Cancer Association in Sweden.

\section{Availability of data and materials}


The datasets used in the current study are available from the corresponding author on reasonable request.

\section{Authors' contributions}

PCL planned the study and collected data. NP analyzed the data and contributed in manuscript writing. All authors read and approved the final manuscript.

\section{Conflict of interest}

The authors declare that they have no competing interests.

\section{Ethics approval and consent to participate}

This study was approved by the Ethics Committee at Uppsala-Örebro, Sweden (dnr 2012/385). All participants received information about the purpose of the study and were assured anonymity and confidentiality before signing a consent form. Informed consent was obtained from all participants in the study.

\section{Consent for publication}

Not applicable

\section{References}

1. Stewart BW, Wild CP, editors (2014) World cancer report 2014 Lyon: International Agency for Research on Cancer

2. Christensen H, Marck DE (2017) The efficacy of mindfulness based stress reduction (MBSR) for decreasing anxiety and depression among breast cancer survivors. Sch Physician Assist Stud $16: 213$

3. The national board of health and welfare (2010) Cancer incidence in Sweden 2009. Stockholm: The national board of health and welfare

4. The national board of health and welfare (2018) Statistics on cancer incidence 2017. Stockholm: The national board of health and welfare ISSN 1401-2016

5. Tryggcadoittir L, Gislum M, Bray F, Klint A, Hakulinen T, Storm HH, Engholm G (2010) Trends in survival of patients diagnosed with breast cancer in the Nordic countries 1964-2003 followed up to the end of 2006. Acta Oncol 49:624-631

6. Jorgensen L, Garne JP, Sogaard M, Laursen BS (2015) The experience of distress in relation to surgical treatment and care for breast cancer: an interview study. Eur J Oncol Nurs 19(6):612-8

7. Tsaras K, Papathanasiou IV, Mitsi D, Veneti A, Kelesi M, Zyga S, Fradelos EC (2018) Assessment of depression and anxiety in breast cancer patients: prevalence and associated factors. Asian Pac $J$ Cancer Prev 19(6):1661-1669 
8. Daldoul A, Khechine W, Bhiri H, Ammar N, Bouriga R, Krir MW, Soltani S, Zoular O, Rhim MS, Bouslah, S, Dimassi S, Abbess I, Saidani Z, Zaied S (2018) Factors predictive of quality of life among breast cancer patients. Asian Pac J Cancer Prev 19(6):1671-1675

9. Arvidsdotter T, Marklund B, Kylen S, Taft C, Ekman I (2016) Understanding persons with psychological distress in primary health care. Scand J Caring Sci 30:687-694

10. Knobf T (2007) Psychological responses in breast cancer survivors. Semin Oncol Nurs 23:71-83

11. Walker J, Hansen CH, Martin P, Symeonides S, Ramessur R, Murray G, Sharpe M (2014) Prevalence, associations, and adequacy of treatment of major depression in patients with cancer: a crosssectional analysis of routinely collected clinical data. Lancet Psychiatry 1:343-350

12. Nakatani Y, Iwamitsu Y, Kuranami M, Okazaki S, Yamamoto K, Watanabe M, Miyaoka H (2013) Predictors of psychological distress in breast cancer patients after surgery. Kitasato Med J 43:49-56

13. Hoyer M, Johansson B, Nordin K, Bergkvist L, Ahlgren J, Lidin-Lindqvist A, Lambe M, Lampic C (2011) Health-related quality of life among women with breast cancer - a population-based study. Acta Oncologica 50(7):1015-1026

14. World Health Organisation Quality of Life Group (1994) The development of the World Health OrganisationQuality of Life assessment instrument (The WHOQOL). In: Orley, J. \& Kuyken, W. (eds.).Quality of life assessment: international perspectives, pp. 41-60. Berlin:Springer-Verlag, Berlin.

15. Montazeri A (2008) Health-related quality of life in breast cancer patients: a bibliographic review if the literature from 1974 to 2007. J Exp Clin Cancer Res 16:1141-1150

16. Debess J, Riis J, Pedersen L, Ewertz M (2009) Cognitive function and quality of life after surgery for early breast cancer in North Jutland, Denmark. Acta Oncol 48:532-540

17. Disipio T, Hayes S, Newman B, Janda M (2008) Health-related quality of life 18 months after breast cancer: Comparison with the general population of Queensland, Australia. Support Care Cancer 16:1141-1150

18. Eaker S, Halmin M, Bellocco R, Bergkvist L, Ahlgren J, Holmberg L, Lambe M (2009) Social differences in breast cancer survival in relation to patient management within a National Health Care System (SWEDEN). Int J Cancer 124:180-187

19. Sharma N, Purkayastha A (2017) Factors affecting quality of life in breast cancer patients: a descriptive and cross-sectional study with review of literature. J Midlife Health 8(2):75-83

20. Papathanasiou IV, Kelepouris K, Valari C, Papagiannis D, Tzavella F, Kourkouta L, Tsaras K, Fradelos EC (2020) Depression, anxiety and stress among patients with hematological malignancies and the association with quality of life: a cross-sectional study. Med Pharm Rep 93(1):62-68

21. Ng CG, Mohamed S, Kaur K, Sulaiman AH, Zainal NZ, Taib NA, and MyBCC Study group (2017) Perceived distress and its association with depression and anxiety in breast cancer patients. Plos One 12:e0172975

22. Zigmond AS, Snaith RP (1983) The Hospital Anxiety and Depression Scale. Acta Psychiatrica Scandinavica 67(6):360-370. Doi:10.1111/j.1600-0447.1983.tb09716.x 
23. Sprangers MA, Groenvold M, Arraras JI, Franklin J, te Velde A, Muller M, Franzini L, Williams A, de Haes HC, Hopwood P, Cull A, Aaronson NK (1996) The European Organization for research and treatment of cancer breast cancer-specific quality-of-life questionnaire module: first results from a three-country field study. J Clin Oncol 14:2756-2768

24. Fayers PM, Machin D (2000) Quality of life: assessment, analysis and interpretation. J Wiley \& Sons Ltd, Chichester ISBN: 0-471-96861-7

25. Suttorp MM, Siegerink B, Jager KJ, Zoccali C, Dekker FW (2015) Graphical presentation of confounding in directed acyclic graphs. Nephrol Dial Transplant 30:1418-23. doi:10.1093/ndt/gfu325

26. Boing L, Pereira GS, de Araujo CC, Sperandio FF, Loch MG, Bergmann A, Borgatto AF, Guimaraes AC (2019) Factors associated with depression symptoms in women after breast cancer. Revista de Saude Publica 53(30)

27. Akel R, Darsa HE, Anouti B, Mukherji D, Temraz S, Raslan R, Tfayli A, Assi, H (2017) Anxiety, Depression and Quality of Life in Breast Cancer Patients in the Levant. Asian Pacific J Cancer Prevent 18(10):2809-2816

28. Srivastava V, Ansari MA, Kumar A, Shah AG, Meena RK, Sevach P, Singh OP (2016) Study of anxiety and depression among breast cancer patients from north India. Clinical Psychiat 2:1(4)

29. Ando N, Iwamitsu Y, Kuranami M, Okazaki S, Nakatani Y, Yamamoto K, Watanabe M, Miyaoka H (2011) Predictors of psychological distress after diagnosis in breast cancer patients and patients with benign breast problems. Psychosomatics 52:56-64

30. Linden W, Vodermaier A, MacKenzie R, Grieg D (2012) Anxiety and depression after cancer diagnosis: Prevalence Rate by cancer type, gender, and age. J Affect Disord 141:343-351

31. Bukovic D, Fajdic J, Hrgovic Z, Kaufmann M, Hojsak I, Stanceric T (2005) Sexual dysfunction in breast cancer survivors. Onkologie 28(1):29-34

32. Drapeau A, Merchand A, Beaulieu-Prevost D (2012) Epidemiology of psychological distress. In Mental IIInesses - Understanding, Prediction, and Control. (LAbate PL. ed.) In Tech. Rijeka 155-34

33. Marchand A, Demers A, Derand P (2015) Do occupation and work conditions really matter? A longitudinal analysis of psychological distress experiences among Canadian workers. Soc Health Illness 27:602-27

34. Boswell EN, Dizon DS (2015) Breast cancer and sexual function. Transl Androl Urol 4(2):160-168

35. Pinto AC, de Azambuja E (2011) Improving quality of life after breast cancer: Dealing with symptoms. Maturitas 70:343-8

36. Poon W, Wang H (2010) Analysis of ordinal categorical data with misclassification. British J Math Stat Psychol 63(1):17-42

\section{Figures}


(a)
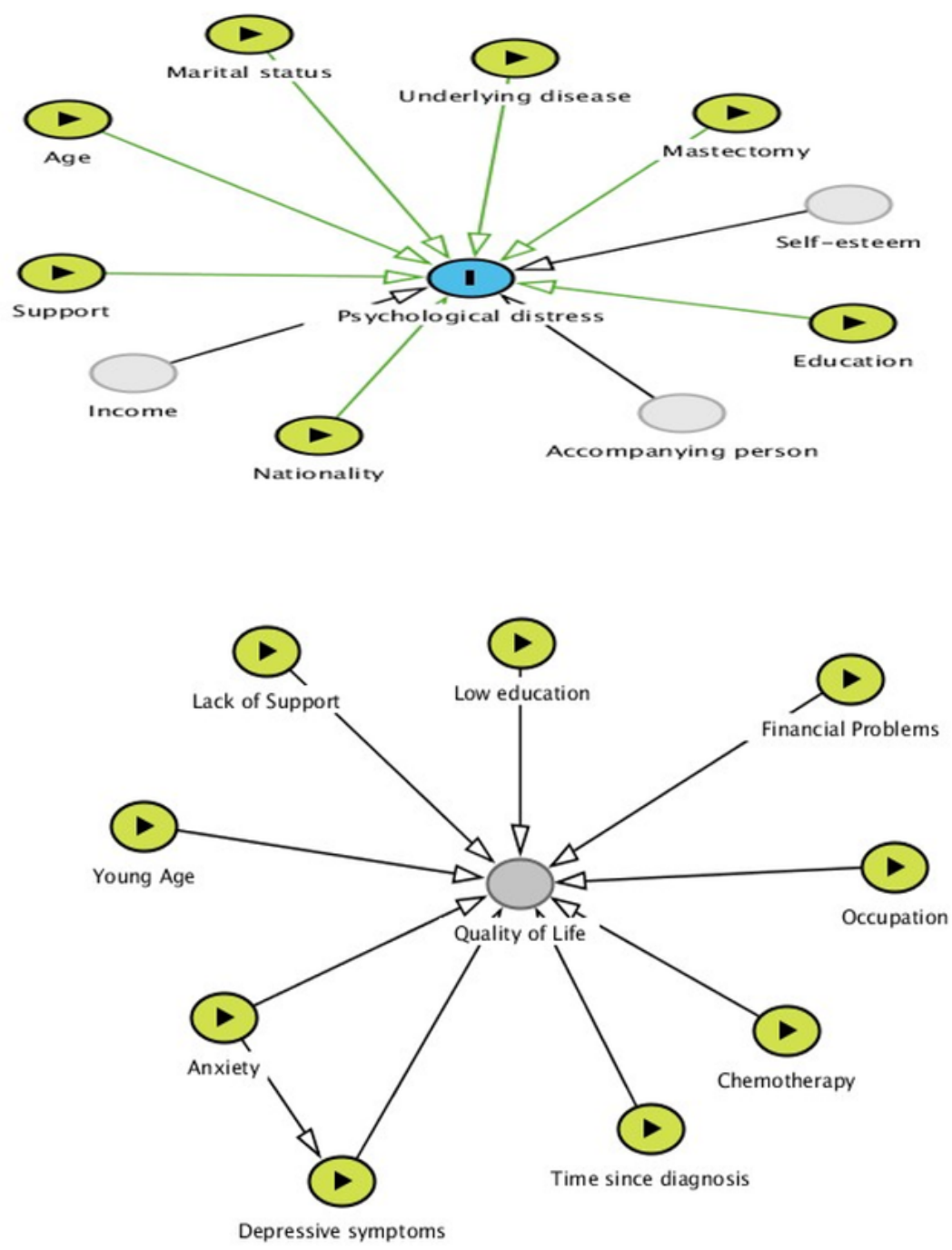

(b)

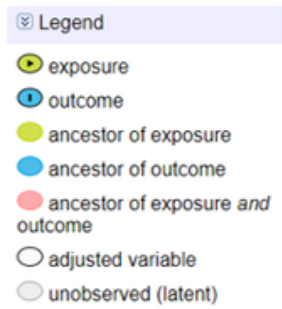

Figure 1

Directed acyclic graphs for possible risk factors associated with (a) psychological distress, and (b) quality of life among cancer patients 